\title{
The Network of Brain Areas Involved in the Motion Aftereffect
}

\author{
J. G. Taylor, ${ }^{*} \dagger$ N. Schmitz, ${ }^{*}+$ K. Ziemons, ${ }^{*}$ M.-L. Grosse-Ruyken, ${ }^{*}$ O. Gruber, ${ }^{*}$ \\ H.-W. Mueller-Gaertner, $*$ \$ and N. J. Shah* \\ *Institute of Medicine, Research Centre Juelich, D-52425 Juelich, Germany; †University Hospital, Heinrich-Heine University, \\ Duesseldorf, Germany; and †Department of Mathematics, King's College, Strand, London WC2R 2LS, United Kingdom
}

Received April 19, 1999

\begin{abstract}
A network of brain areas is expected to be involved in supporting the motion aftereffect. The most active components of this network were determined by means of an fMRI study of nine subjects exposed to a visual stimulus of moving bars producing the effect. Across the subjects, common areas were identified during various stages of the effect, as well as networks of areas specific to a single stage. In addition to the well-known motion-sensitive area MT the prefrontal brain areas BA44 and 47 and the cingulate gyrus, as well as posterior sites such as BA37 and BA40, were important components during the period of the motion aftereffect experience. They appear to be involved in control circuitry for selecting which of a number of processing styles is appropriate. The experimental fMRI results of the activation levels and their time courses for the various areas are explored. Correlation analysis shows that there are effectively two separate and weakly coupled networks involved in the total process. Implications of the results for awareness of the effect itself are briefly considered in the final discussion. 2000 Academic Press
\end{abstract}

\section{INTRODUCTION}

The motion aftereffect (MAE) occurs after adaptation to a moving scene, when ensuing observation of a stationary scene causes experience of motion in the opposite direction. Considerable study, dating back to the previous century, has been made of the psychophysical nature of the effect (Adams, 1884; Wohlgemuth, 1911); this has been updated by analysis of more detailed psychophysical features of the phenomenon (Mather et al., 1998). Recently the initial brain region involved in the experience has been uncovered. There is evidence (Tootell et al., 1995a) that the time course of activation of area MT in humans during the experience of MAE mirrors that observed psychophysically. That is not to say that there are no other areas in the cortex also importantly involved in helping support the experi- ence; the purpose of this study is to look for such areas and attempt to assess their contribution.

There is also the phenomenal experience itself. From the results of Tootell et al. (1995a) it might be suspected that this is mainly supported by area MT. However, these results do not prove that the phenomenal experience of MAE occurs specifically in that area, especially since it has been proposed that phenomenal experience itself especially involves activation of posterior working memory/multimodal sites and requires a suitable period of time to achieve breakthrough to awareness in competition against already existing experienced activity (Taylor, 1997).

Preliminary experimental results have already been presented showing that indeed the experience of the MAE is not perfectly correlated with activity in MT (He et al., 1998). These studies used a period of blank screen, just after the movement, when no MAE experience occurred. Re-presentation of the static scene recreated the MAE effect. However, the activity in MT was observed to continue unabated through the "white" period on the screen, so the correlation with the MAE experience was destroyed.

The purpose of the present fMRI study is thus twofold:

(a) to replicate the results of Tootell et al. (1995a) and

(b) to search for the total network of brain sites involved in MAE.

In general, then, the main thrust of the experimental part of the study is to search for the total set of brain regions in which activity specifically related to MAE appears and study their character. This involves looking at motion-sensitive areas involved in the total phenomenon.

We use fMRI for the study, rather than PET, for a number of reasons: (a) there is no radioactivity involved, so repeat experiments can be done on the same volunteer to optimize the experiment, (b) longitudinal studies can therefore be performed, (c) even singlesubject experiments produce significant results, so that the robustness of results from a group study can be ascertained across the subjects, and (d) determination 
of the detailed timing of the emergence and continuation of activity time locked to the motion aftereffect period can be achieved. The possibility of observing such time courses depends crucially on using a suitable paradigm to enable the timing to be specified. If we use a moving stimulus (in our case a set of moving bars) which ceases its motion at a certain time then we have an offset point from which to time any changes of activity, as well as for the subject to experience the motion aftereffect itself. It is also necessary to compare with activations brought about by exposure of the subject to a control condition, such as the bars moving up and down. For that stimulus there will be no aftereffect. This was the basis of the method used in Tootell et al. (1995a) (although with a different stimulus composed of moving and static rings) and is used more extensively here. We will search for activations, especially in prefrontal sites, which are timed exactly with the offset of bars moving either in one direction (we used only bars moving down in this study) or up and down successively. In addition to MT, any region in which activation commences as the stimulus movement ceases at the end of the motion down period, and then decays over about the expected time for the MAE experience, is a candidate for the network of areas observing the MAE experience itself. The signal after the one-way movement, compared to that in the up-anddown case, gives the net MAE effect. That characteristic is the main signal for which we searched in the fMRI data.

There are also other characteristics of signals expected from regions involved in the overall processing. Thus we expect to see sites involved in analysis of the stationary bars during the period just after the downward motion, used as a testing period to measure the duration of the motion aftereffect experience noted above. This period itself can be split into one during which the MAE is being experienced and one in which there are only static bars, together with the fixation cross (present throughout the whole experiment to prevent confounding eye movements), being observed and experienced. In order to achieve the highest attention during the whole period of exposure of the subjects we did not tell them that they would not experience any MAE after the up-and-down motion period, so there is also the putative MAE period after the up-and-down motion period. We will also consider those areas which are especially active during one or more of downward MAE, putative up-and-down MAE, and Static as well as during either motion period. As part of the data analysis, we used suitable reference vectors which were particularly sensitive to these various parts of the paradigm so as to select the regions activated differentially. It turned out, on further questioning, that different subjects used different strategies. We discuss these individual differences in due course.
Our main findings are that there are some remarkable time courses in both posterior and prefrontal cortical areas which differ significantly across these periods and across the different areas. Some sites (such as in BA 40, 44, or 47 or the cingulate gyrus) are active only during either of the putative MAE periods. Others (also in BA 44 or 47 or 40R) are active only or mainly during downward-caused MAE. Yet others (such as in MT/V5) are active during both motion periods and the downward MAE. These results indicate that there are different networks involved in different stages of the total experience. Some areas have different components involved in different stages and very likely in different networks. We explore later the nature of these various networks and their involvements in the total time course.

The analysis is compounded by the fact that there is considerable variation between the activation time courses across the subjects, both outside the machine in the psychophysical test and inside the bore of the magnet. This range was quite considerable, from 5.4 up to $18.5 \mathrm{~s}$ in the former case and from $4.1 \mathrm{up}$ to $14 \mathrm{~s}$ in the latter. We thus have to note that our results are valid only for the group average; different subjects may use different strategies in checking for the MAE.

The contents of the paper are as follows. After discussing the methods used and our data analysis techniques we present the group results in graphical and tabular form. The following section develops a functional network for achieving the task at the group level. The paper concludes with a discussion of the results and their implications for analysis of the awareness of MAE itself.

\section{METHODS}

Nine healthy subjects (six men, three women), without any history of neurological or audiological illness, were studied. Subjects gave informed, written consent following a full explanation of the nature and risks of the research, according to a protocol approved by the Ethics Committee of the Heinrich-Heine University of Duesseldorf. High-resolution, T1-weighted anatomical image of the entire brain were obtained in 3D using the MP-RAGE (magnetization-prepared, rapid-acquisition gradient echo) pulse sequence with the following parameters: TR (repetition time) $=11.4 \mathrm{~ms}$; TE (echo time $)=4.4 \mathrm{~ms}, \theta$ (flip angle) $15^{\circ} ; 1$ excitation; FOV (field of view) $230 \mathrm{~mm}$; matrix $200 \times 256 ; 128$ sagittal slices with 1.41-mm slice thickness. Echo planar imaging (EPI) was performed on a Magnetom Vision 1.5-T scanner (Siemens Medical Systems GmbH, Erlangen, Germany) equipped with a gradient booster system; the standard radiofrequency head coil was used for transmission and reception. Pulse sequence parameters were as follows: gradient echo EPI; TR $=3 \mathrm{~s}$; TE $=66$ 
$\mathrm{ms}$; FOV $200 \times 200 \mathrm{~mm} ; \theta 90^{\circ}$, matrix size $64 \times 64$; pixel size $3.125 \times 3.125 \mathrm{~mm}$; 16 slices; slice thickness $3.0 \mathrm{~mm}$; interslice gap $0.3 \mathrm{~mm}$. We denote the full set of 16 slices as a scan.

The fMRI paradigm consisted of a preceding baseline of $30 \mathrm{~s}$ (10 × (the TR of) $3 \mathrm{~s}$ for each scan) followed by four repetitions of a module composed of two different 21-s periods of moving stimuli (each involving seven scans) and two interspersed 21-s periods of static stimuli (each of seven scans). In order to allow the signal to reach a steady state, the first three scans from the initial baseline were discarded.

Each experiment was started only after a prior determination of the extent of MAE for each subject before entering the magnet. They sat facing a screen on which the program used for the main experiment was projected and which recorded the extent of their MAE experience by means of their pressing a timing button when they considered their MAE experience finished. The same fixation cross was present throughout the stimulus period as used in the magnet, and the subjects were requested to fixate throughout this period. No attempt was made to detect eye movement during this psychophysical study, but subjects did not report any difficulty of fixation throughout the study.

The main experimental time course in the experiment consisted of an initial baseline of 10 scans, of which 3 were discarded to allow for approach of the signal to steady state, followed by four repetitions of a moving-static-moving-static module of 7 scans each. The time course of the stimuli and their characteristics are shown in Fig. 1.

Stimuli consisted of moving/static bars developed using the MEL software system (MEL Professional, 1993), which allowed the continuous display of $21 \mathrm{~s}$ of movement of the bars in the up, the down, or the up/down directions or of static bars for $21 \mathrm{~s}$. Only the downward moving bars (being shown during the period which we termed DO) were used in our experiments to induce the motion aftereffect, in addition to the control of the up/down movements and the static bars for the period of the experience itself. The 21-s periods of the presentation of the moving bars (either downward or up and down) were interspersed with $21 \mathrm{~s}$ of static bars (during the period termed Static). During this period the subjects viewed the stationary bars and reported the temporal extent of the MAE experience. This could be an experience of MAE either just after the cessation of the downward motion period (which we term MAEDO) or at cessation of the up-and-down motion period (termed MAE-BO). In interspersed periods were movement periods in which the bars moved up and then down (in the period termed BO) as a control condition (as shown in Fig. 1). The Static period is thus divided into a motion aftereffect period and a rest period. Thus after the motion-DO period, Static $=$ MAE-DO + Rest, and after the motion-BO period, Static $=$ MAE-BO + Rest. The length of the MAE-DO and the MAE-BO periods was taken, from the data in the scanner, to be three scans (see below and Table 1), so the Rest periods are both four scans (as shown in Fig. 1). The stimuli were projected onto a screen fitting closely into the bore of the magnet (so as to avoid images moving in several directions). Subjects were scanned with room lights completely dimmed.

Before the experiment each subject was asked to record the extent of his or her MAE by pressing the button at the moment the experience ceased. This was asked for at the end of both of the movement periods for the bars, so for both the up and the down or both movements. The extent of a subject's experience of MAE at the end of these periods while he or she was in the magnet was automatically recorded by means of the button-press system (as used in the psychophysical experiments mentioned earlier). This used a light fiber system and LEDs; the buttons cause a break in the transmitted light and this is detected by electronics which then send a pulse to a PC. The average of the MAE duration period across subjects was used to determine a psychophysical MAE period of $9 \mathrm{~s}$ after either the DO or the BO motion period. This agreed with the earlier results of Tootell et al. (1995a).

\section{DATA ANALYSIS}

Image analysis was performed on a SPARC 20 workstation (Sun Microsystems) using MATLAB (version 4.2c, The Mathworks, Inc., Natick, MA) and SPM967 software (Statistical Parametric Mapping, The Wellcome Department of Neurology, London) (Friston et al., 1994, 1995). The first three images of each time series, during which the MR condition was automatically realigned, were discarded. The remaining volume data sets of each condition were automatically realigned to the first image to correct for head movement between scans. All data sets were motion corrected using the coregister and reslice algorithms in SPM96 (Friston et al., 1994, 1995). Pixels were considered statistically significant if the correlation of the pixel time series with a delayed box-car reference function exceeded a $z$ score of at least 3.09 (corrected $P<0.001$ ). Regions were selected by using reference contrasts designed to pick out areas sensitive specifically for motion and the MAE or only for the MAE, as part of SPM-96b (Friston et al., 1995).

Six contrasts proved most effective. These used the seven conditions occurring during one or another of the DO, BO, MAE-DO, or MAE-BO periods. More specifically the conditions were (with the number of scans in parentheses)

1. Baseline (3)

2. $\mathrm{DO}(7)$ 


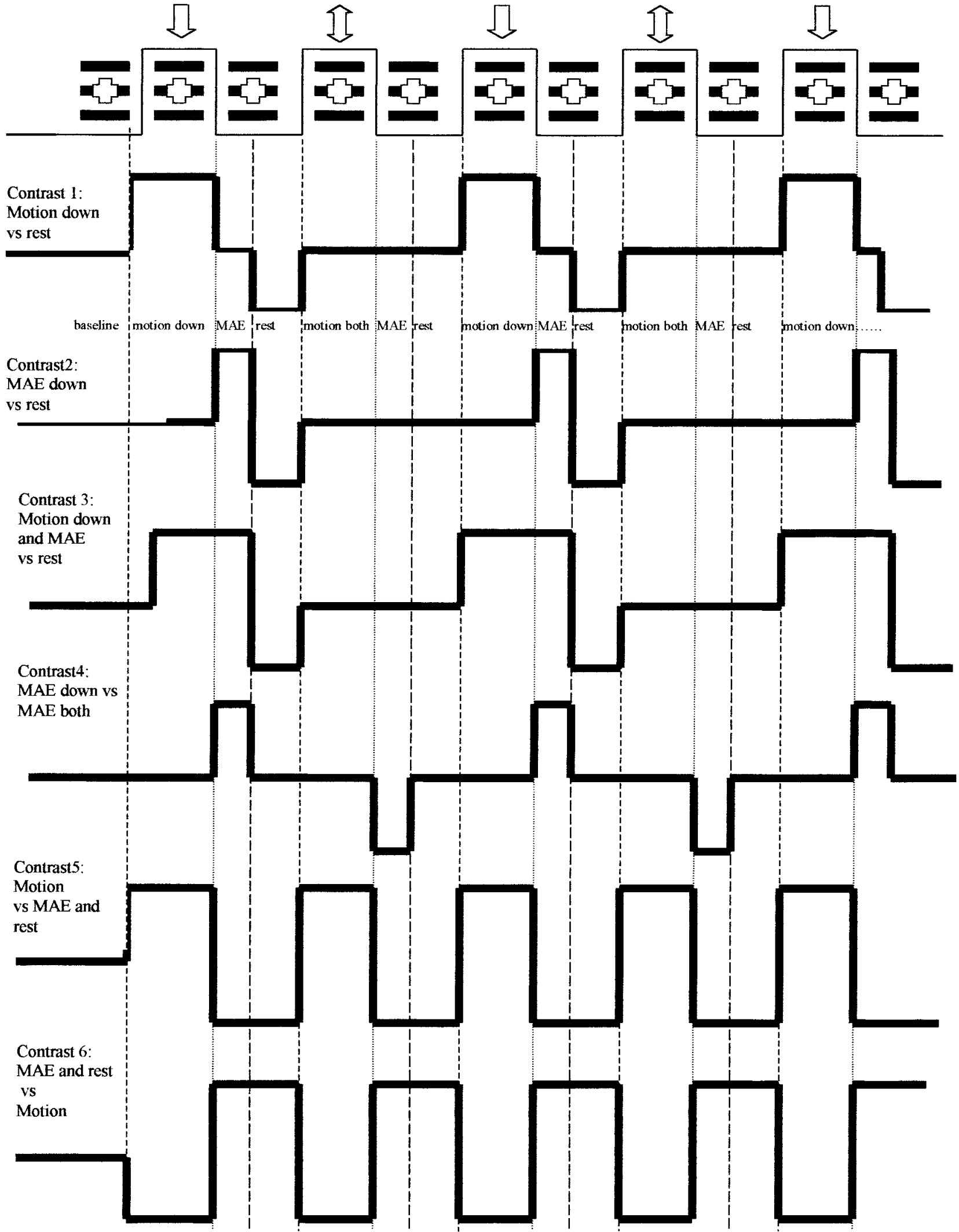

FIG. 1. Time course of the stimuli and their characteristics, together with time courses of the six contrasts. 

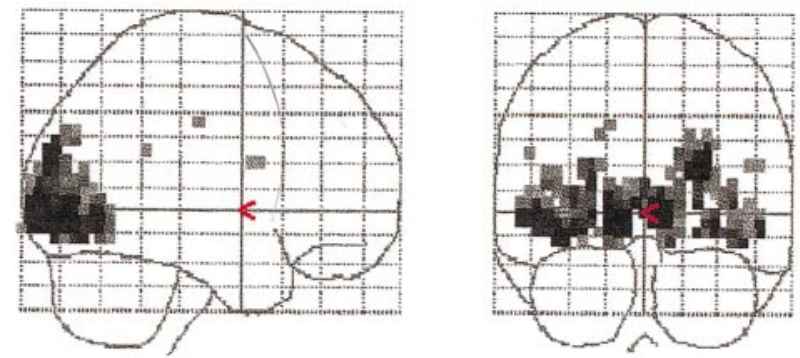

Motion down vs static, contrastl
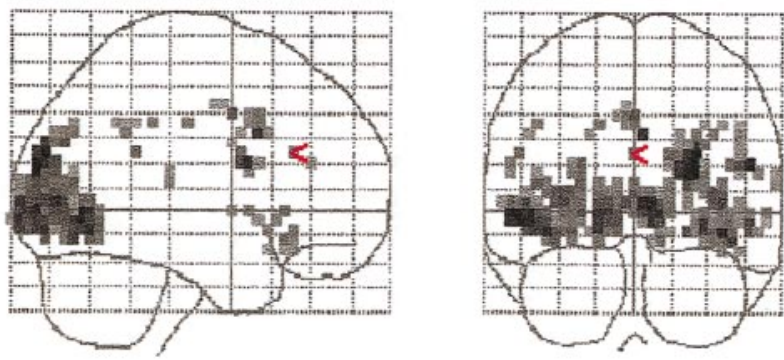

Motion and MAE vs static, contrast 3
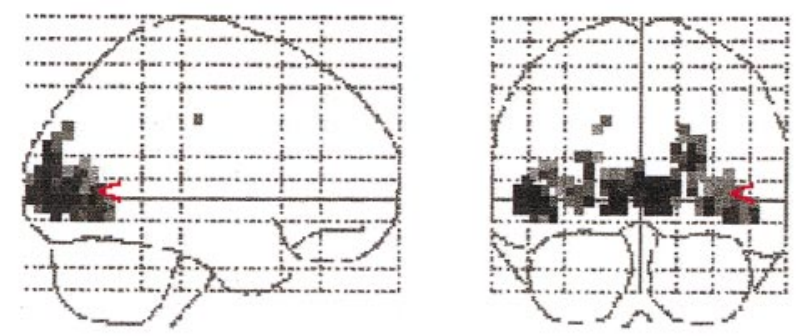

Motion only, contrast 5
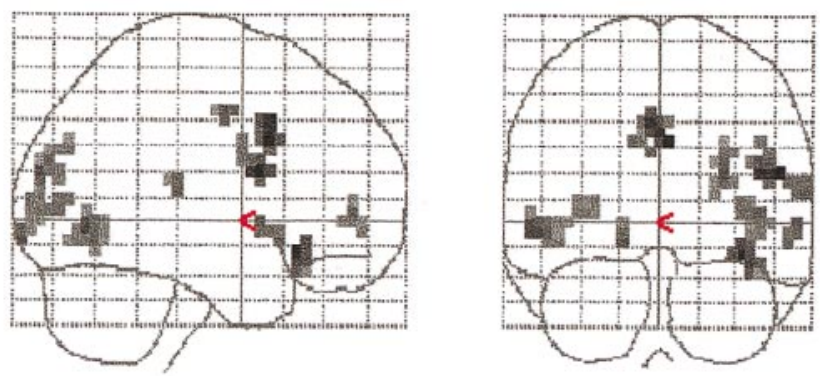

MAE down vs static, contrast 2
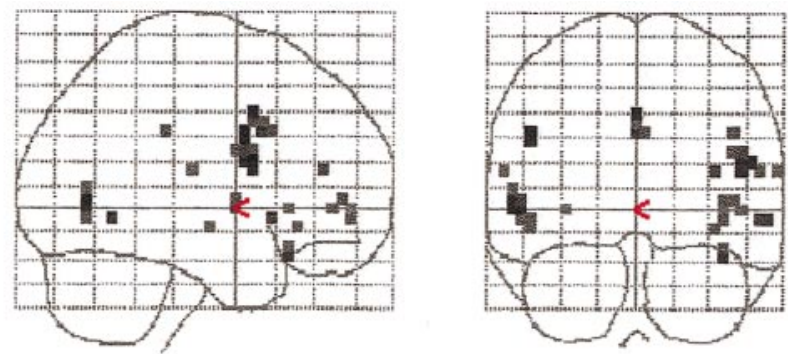

MAE of down vs MAE of both, contrast4
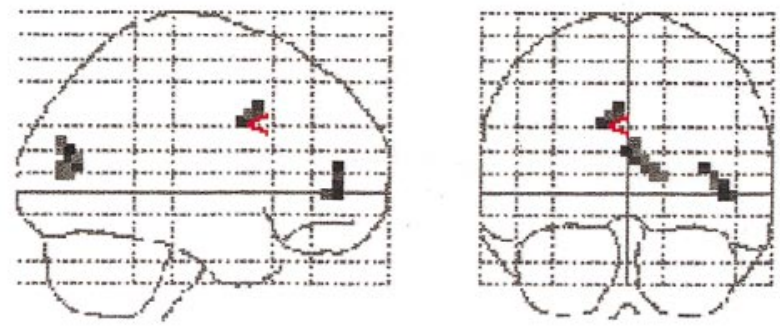

Static only, contrast 6

FIG. 2. Glass brains of the results of the group study for each of the six contrasts.
3. MAE-DO (3)
4. Static (4)
5. $\mathrm{BO}(7)$
6. MAE-BO (3)
7. Static (4)

Thus

Contrast 1: DO vs Static

Contrast 2: MAE-DO vs Static

Contrast 3: DO + MAE-DO vs Static

Contrast 4: MAE-DO vs MAE-BO

Contrast 5: DO + BO vs MAE-DO + MAE-BO + Static (motion only vs static, including motion aftereffect)

Contrast 6: MAE-DO + MAE-BO + Static vs BO + DO (static, including motion aftereffect vs motion)

In terms of the vectorial representations of the conditions, the contrast vectors have the values $(0,1,0$, $-1,0,0,0),(0,0,1,-1,0,0,0),(0,1,1,-2,0,0,0),(0,0$, $1,0,0,-1,0),(0,2,-1,-1,2,-1,-1)$, and $(0,-2,1,1$, $-2,1,1)$, where the initial zero component in each vector of contrasts corresponds to the discarded first three scans.

The resulting contrasts are then fitted to the time courses of the four active-rest-active-rest repeats in a least-mean squares manner, using independent delayed box-car vectors for each of the seven relevant conditions, with the same parameters in the fit to the data over the four repeats.

\section{RESULTS}

Psychophysical Values

We present in Table 1 the MAE times taken before and during the experiment in the magnet. It is seen that there is considerable variability across subjects 


\section{TABLE 1}

MAE Psychophysical Time Datasheet

\begin{tabular}{cccc}
\hline & $\begin{array}{c}\text { Psychophysical } \\
\text { MAE } \\
\text { Subject }\end{array}$ & $\begin{array}{c}\text { MAE in } \\
\text { magnet } \\
\text { after DO (s) }\end{array}$ & $\begin{array}{c}\text { MAE in } \\
\text { magnet } \\
\text { after BO (s) }\end{array}$ \\
\hline 1 & 14.3 & 4.2 & 0.0 \\
90 & 13.3 & 4.1 & 1.6 \\
89 & 7.5 & 6.1 & 1.4 \\
88 & 11.8 & 6.7 & 0.0 \\
87 & 8.7 & 5.9 & 1.1 \\
69 & 12.3 & 6.0 & 1.8 \\
86 & 8.6 & 5.1 & 1.5 \\
70 & 5.4 & 4.5 & 0.0 \\
71 & 18.5 & 14.0 & 0.0 \\
Mean & 8.1 & 6.3 & 0.8 \\
SD & 4.0 & 3.0 & 0.8 \\
\hline
\end{tabular}

Note. The values entered in the first column were obtained from subjects before entering the magnet. The values in the second column were the subjects' response after experiencing the DO stimulus (motion of the bars in the downward direction only) and in the third column after the BO stimulus (when the bars moved first upward and then downward).

(which we had also found in our preliminary studies) as well as in the comparison between the times measured outside the magnet prior to the experiment and that in the magnet during the fMRI runs. The former of these variations is to be expected although we were surprised to discover some subjects for whom there was almost no MAE experienced even outside the magnet and under normal conditions (these were not included in our study). There was also an important variation of size of MAE across subjects between testing for MAE outside or testing inside the magnet. Such a difference may be due to the subjects' lying down when in the magnet. It may also arise from the changed conditions in the magnet, especially with increased noise. We do not think it is due to the reduced size or intensity of the stimulus in the magnet compared to outside it, although there may have been some reduction. However, the stimulus presentation in the magnet was optimized for intensity and sharpness. Nor do we consider the reduction due to lessened attention in the scanner; none of the subjects reported any such experience, despite results on the effects of attention on the MAE duration (Chaudhuri, 1990). The experiment is being repeated with $\mathrm{PET}$ with the subjects sitting upright in a chair in order to check if the specific conditions inside the magnet may explain these effects.

\section{fMRI Analysis: Group Study}

The results of the group study were analyzed using the six different contrasts mentioned above. In general, activity associated with motion processing (including the MAE period) was observed in visual cortex, MT, and several anterior regions, while that specifically associ- ated with MAE was observed in numerous posterior and anterior cortical sites. This is shown for the different contrasts from the group study in the glass brains of Fig. 2. Regions active under the six contrasts, and their $z$ scores and Tailairach and Tourneaux (1988) coordinates, are shown in Table 2.

The sites of the different regions passing the threshold $z=3.09(P=0.001)$ are shown in the glass brains of Fig. 2. A general trend of increasing anterior activation and reducing posterior activation is clear in proceeding from contrasts 1 and 5 to contrast 3 , then contrast 2 , and finally contrast 4 . This corresponds to increasing "anteriorization" involved with the MAE periods MAE-DO and MAE-BO, which are increasingly being singled out by the contrasts considered, as seen from the contrasts under Data Analysis. Thus contrasts 1 and 5 are involved only with the motion periods compared to the static periods, while at the other extreme contrasts 2 and 4 concentrate on the MAE periods. Contrast 3 is intermediate. Finally contrast 6 is mainly concerned with processing of the static stimuli, and interestingly involves both extrastriate visual cortex and cingulate as well as BA10. The nature of the time courses in sites selected by contrasts 1 to 5 will be considered together next; those arising from use of contrast 6 will be discussed separately, since they emphasize regions involved mainly with processing static features.

We add that we have labeled the brain areas containing the active sites detected by our data analysis using the areas specified in the Talairach and Tournoux atlas; this may be controversial since some of the areas have undefined boundaries, but we do not wish to enter into such controversies here; we can only refer those concerned to the sites on the atlas (Talairach and Tournoux, 1988). The corresponding gyri at which these points are sited are also included in Table 2. MT is outside this labeling; for that we have used Tootell et al. (1995b).

We will now present and analyze the time courses of a selected set of the regions noted in Table 2; these have all been selected by contrast 4 , so automatically have more activity in MAE-DO than in MAE-BO.

\section{Time Course of $M T$}

This time course is shown in Fig. 3a. The time course shows activation which follows the movement of the stimulus. This is to be seen from the continuous regression curve of the reference function defined from the conditions. There are four activation curves arising from the scans taken during each of the down periods, interspersed with slightly narrower and lower activation peaks from scans during the up-and-down motion 
TABLE 2

Coordinates of Regions of Activation in Different Brain Areas Detected Under the Six Different Contrasts for the Group Study and as Shown in the Glass Brains in Fig. 2

\begin{tabular}{|c|c|c|c|c|c|c|c|c|c|c|c|c|}
\hline \multirow{2}{*}{\multicolumn{2}{|c|}{$\begin{array}{l}\text { Contrast and } \\
\text { coordinates }\end{array}$}} & \multicolumn{11}{|c|}{ Brain area } \\
\hline & & 17/18 right & $17 / 18$ left & 18 right & & 18 left & $18 / 19$ right & 19 right & 19 left & MT right & MT left & $\mathrm{CG}$ \\
\hline (1) & DO vs Static & $\begin{array}{l}12-92-8 \\
z \text { score } 4.9 \\
\text { GL }\end{array}$ & $\begin{array}{l}-16-96-8 \\
z \text { score } 5.4 \\
\text { GL-GF }\end{array}$ & $\begin{array}{l}28-92-8 \\
z \text { score } 4.8 \\
\text { GF }\end{array}$ & $\begin{array}{l}12-80-4 \\
z \text { score } 6.2 \\
\text { GL }\end{array}$ & $\begin{array}{l}-28-92-4 \\
z \text { score } 4.4 \\
\text { GOi }\end{array}$ & & & $\begin{array}{l}-12-76-4 \\
z \text { score } 7.4 \\
\text { GL }\end{array}$ & $\begin{array}{l}48-640 \\
z \text { score } 3.1 \\
\text { GTi }\end{array}$ & $\begin{array}{l}-52-68-4 \\
z \text { score } 6.7 \\
\text { GOm }\end{array}$ & \\
\hline (2) & $\begin{array}{l}\text { MAE of DO vs } \\
\text { Static }\end{array}$ & & & & & & & $\begin{array}{l}32-8028 \\
z \text { score } 3.7 \\
\text { GO }\end{array}$ & $\begin{array}{l}-32-888 \\
z \text { score } 3.4 \\
\text { GOm }\end{array}$ & & $\begin{array}{l}-48-680 \\
z \text { score } 3.5 \\
\text { GTi }\end{array}$ & $\begin{array}{l}-41228 \\
z \text { score } 4.5 \\
\text { CG }\end{array}$ \\
\hline (3) & $\begin{array}{l}\text { Mae DO and } \\
\text { motion DO vs } \\
\text { Static }\end{array}$ & $\begin{array}{l}12-92-8 \\
z \text { score } 5.5 \\
\text { GL }\end{array}$ & $\begin{array}{l}-16-98-8 \\
z \text { score } 5.9 \\
\text { GL-GF }\end{array}$ & $\begin{array}{l}28-92-8 \\
z \text { score } 4.1 \\
\text { GF }\end{array}$ & & $\begin{array}{l}-28-92-8 \\
z \text { score } 4.0 \\
\text { GOi }\end{array}$ & $\begin{array}{l}24-8020 \\
z \text { score } 6.6 \\
\mathrm{Cu}\end{array}$ & & $\begin{array}{l}-24-8820 \\
z \text { score } 5.8 \\
\text { GO }\end{array}$ & & $\begin{array}{l}-52-68-4 \\
z \text { score } 6.7 \\
\text { GOm }\end{array}$ & $\begin{array}{l}41232 \\
z \text { score } 6.1 \\
\text { CG }\end{array}$ \\
\hline (4) & $\begin{array}{l}\text { Mae DO vs } \\
\text { Mae BO }\end{array}$ & & & & & & & & & & $\begin{array}{l}-52-68-4 \\
z \text { score } 3.3 \\
\text { GOm }\end{array}$ & $\begin{array}{l}01232 \\
z \text { score } 3.8 \\
\text { CG }\end{array}$ \\
\hline (5) & $\begin{array}{l}\text { Motion of all } \\
\text { conditions }\end{array}$ & & & & & & & & & & $\begin{array}{l}-52-68-4 \\
z \text { score } 7.3 \\
\text { GOm }\end{array}$ & $\begin{array}{l}41228 \\
z \text { score } 3.9 \\
\text { CG }\end{array}$ \\
\hline (6) & Static only & & & & & & & $\begin{array}{l}4-8024 \\
z \text { score } 3.9 \\
\text { GO-Cu }\end{array}$ & & & & $\begin{array}{l}-8832 \\
z \text { score } 5.0 \\
\text { CG }\end{array}$ \\
\hline (1) & DO vs Static & 44 right & 44 left & 40 right & & 40 left & 37 right & 46 right & 47 right & & 22 right & 10 right \\
\hline (2) & $\begin{array}{l}\text { MAE of DO vs } \\
\text { Static }\end{array}$ & $\begin{array}{l}56816 \\
z \text { score } 4.5 \\
\text { GFi }\end{array}$ & & & & & & & $\begin{array}{l}3228-12 \\
z \text { score } 4.9 \\
\text { GFi }\end{array}$ & & $\begin{array}{l}6012-4 \\
z \text { score } 4.4 \\
\text { GTs }\end{array}$ & $\begin{array}{l}48440 \\
z \text { score } 3.7 \\
\text { GFm }\end{array}$ \\
\hline (3) & $\begin{array}{l}\text { Mae DO and } \\
\text { motion DO vs } \\
\text { Static }\end{array}$ & $\begin{array}{l}48820 \\
z \text { score } 4.0 \\
\text { GFi }\end{array}$ & & & & & & & $\begin{array}{l}3228-12 \\
z \text { score } 3.6 \\
\text { GFi }\end{array}$ & & & \\
\hline (4) & $\begin{array}{l}\text { Mae DO vs } \\
\text { Mae BO }\end{array}$ & $\begin{array}{l}48428 \\
z \text { score } 3.6 \\
\text { GFi }\end{array}$ & $\begin{array}{l}-48428 \\
z \text { score } 4.3 \\
\text { GFi }\end{array}$ & $\begin{array}{l}44-3232 \\
z \text { score } 3.5 \\
\text { LPi }\end{array}$ & & $\begin{array}{l}-60-2016 \\
z \text { score } 3.3 \\
\text { GPoC }\end{array}$ & $\begin{array}{l}60-56-4 \\
z \text { score } 3.9 \\
\text { GTi }\end{array}$ & $\begin{array}{l}364016 \\
z \text { score } 3.7 \\
\text { GFi }\end{array}$ & $\begin{array}{l}3628-8 \\
z \text { score } 3.4 \\
\text { GFi }\end{array}$ & & & \\
\hline (5) & $\begin{array}{l}\text { Motion of all } \\
\text { conditions }\end{array}$ & $\begin{array}{l}48816 \\
z \text { score } 3.1 \\
\text { GFi }\end{array}$ & $\begin{array}{l}-52424 \\
z \text { score } 3.8 \\
\text { GFi }\end{array}$ & & & & $\begin{array}{l}60-56-4 \\
z \text { score } 3.3 \\
\text { GTi }\end{array}$ & & & & & \\
\hline (6) & Static only & & & & & & & & & & & $\begin{array}{l}364812 \\
z \text { score } 6.1 \\
\text { GFm }\end{array}$ \\
\hline
\end{tabular}

Note. Abbreviations used (Latin): Cu, cuneus; GF, gyrus fusiformis; GL, gyrus lingualis; GFi, gyrus frontalis inferior; GFm, gyrus frontalis medialis; GC, gyrus cinguli; GO, gyri orbitales; GOi, gyrus occipitalis inferior; GOm, gyrus occipitalis medialis; GPoC, gyrus postcentralis; GTi, gyrus temporalis inferior; GTs, gyrus temporalis superior; LPi, lobulus parietalis inferior.

period. All eight of these have onset at the beginning of the relevant motion periods. However, the peaks for the DO period are wider than those for the BO period by 3.5 scans or equivalently by $10.5 \mathrm{~s}$. This corresponds to a delay in cessation of the activation during the MAE period after DO. It signals the presence of the MAE signal with a value of $10.5 \mathrm{~s}$ for its duration. This can be supported by a more careful analysis of the time courses of the peaks in the time series itself arising at the end of the relative motion period. These delays are $3,3,2$, and 3 scans, respectively, so corresponding to an MAE signal lasting for on average 2.75 scans or $8.25 \mathrm{~s}$. This fits closely with the internally reported MAE signal taken during the experiment. It is that agreement which we take as validating the earlier study of Tootell et al. (1995a). Finally we note that there is no hint of MAE after the BO period of motion. The mean value reported by the subjects (given in Table 1) during that period is only $0.8 \mathrm{~s}$, a value below the sensitivity of the machine.

\section{Time Course of BA4OL}

We now move more anterior and dorsal to MT to consider the activation time course in BA40L. This time course is shown in Fig. 3b. The time course shows activation which is linked to the movement of the stimulus but is very different from that of MT. This is to be seen from the continuous regression curve of the reference function defined from the conditions. There are four highly significant activation curves arising from the scans taken just after each of the down periods, interspersed with somewhat lower activation peaks from scans just after the up-and-down motion period. All eight of these have onset precisely at the cessation of the relevant motion period. This area therefore is devoted only to the possible MAE periods, with more activation during the presence of the MAE experience itself (from the greater activity in the MAE-DO period compared to the MAE-BO period).

We noted earlier that there was no evidence for any 

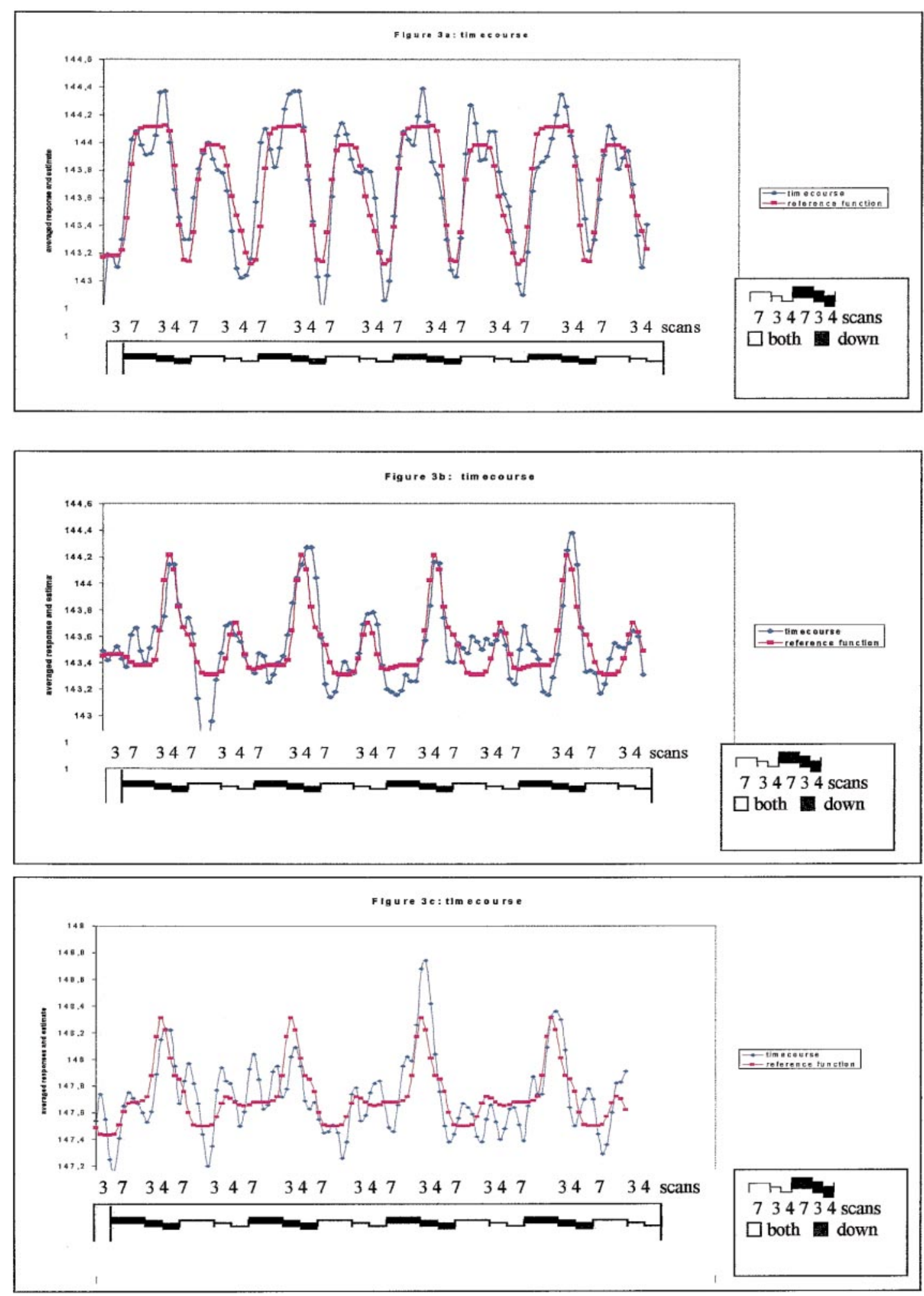

FIG. 3. Time course in the group study of (a) MT under contrast 4 at the point $(-52,-68,-4)$, with $z=4.14$; (b) BA40L under contrast 4 at the point $(-60,-20,16)$, with $z=3.28$; (c) BA40R under contrast 4 at the point $(44,-32,32)$, with $z=3.55$; (d) BA44 under contrast 4 at the point $(48,4,28)$, with $z=3.62$; (e) BA37 under contrast 4 at the point $(60,-56,-4)$, with $z=3.86$; and (f) BA18 under contrast 6 at the point ( 8 , $-76,12)$, with $z=3.61$. 

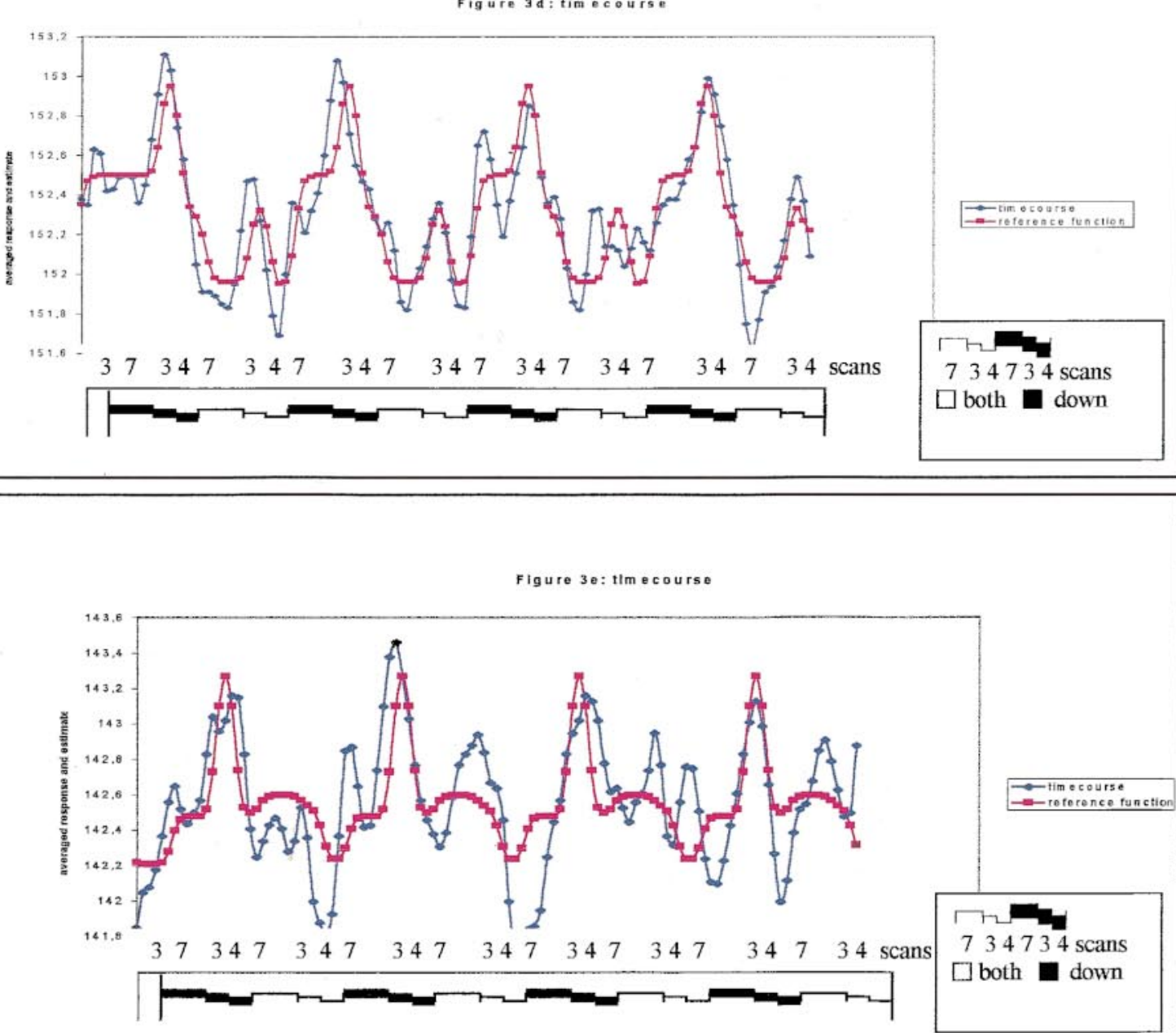

FIG.3-Continued 
sizeable MAE signal during the motion BO period and certainly not of the same level as that during the motion DO period. The signal seen in Fig. 3b during that time is less than half that during the MAE-DO period. Thus BA40L is performing some control function to search for MAE and indicating effects from other regions performing that checking function. It is also involved to a certain extent with generation of the MAE experience itself. We will discuss this aspect later.

We show in Fig. 3c the time course of a pixel from BA40 right. This has a time development similar to that of Fig. $3 \mathrm{~b}$, but there is less evidence than for BA40L of an activation peak during the MAE-BO period. This indicates that the above suggestion of the involvement of BA40 in creating the MAE experience is more strongly supported by the activation time course in this site in BA40, with more of a checking function being performed in BA40L during the MAE-BO period.

\section{Time Course of BA44R}

We now move more anterior to MT to consider the activation time course in BA44. This time course is shown in Fig. 3d. The time course shows activation which is linked to the movement of the stimulus as well as during the MAE period, but is different from that of MT as well as that of BA40, which we have just discussed. This is seen from the continuous regression curve of the reference function defined from the conditions in Fig. 3d. There are four high-activation curves arising from the scans taken in the MAE-DO period just after each of the down periods, interspersed with lower activation peaks from scans during the motion DO. There are also four lower sharp peaks just after the cessation of the motion BO. Thus this area seems to be devoted to several features:

(1) The MAE-DO period.

(2) The MAE-BO period to a lesser extent than the MAE-DO period, but again like BA40L independent of the presence of the MAE activity itself. We noted under Time Course of BA40L that there was no evidence for any sizeable MAE signal during the motion $\mathrm{BO}$ period in BA40R and certainly not of the same level as that during the motion DO period. Thus BA44R is also performing some control function to search for MAE at about the same extent as BA40L.

(3) The motion DO period. This is quite different from the sensitivity of MT to all motion; here BA44 is able to ignore the motion BO input completely.

\section{Time Course of BA37R}

We now consider the activation time course of BA37R. A particular time course is shown in Fig. 3e. The time course shows activations which are linked to the movement of the stimulus but is very different from that of
MT; however, it is closer to that of BA40R, which was discussed above. It demonstrates strong activity during the MAE-DO period. This is to be seen in Fig. 3e from the continuous regression curve of the reference function defined from the conditions: there is clear motion sensitivity in the MAE-DO period, as well as much lower sensitivity in the MAE-BO period. There is the least sensitivity in the rest period after the MAE-BO period. This differentiates it from BA40R, which has least activity in the MAE-DO period.

\section{Time Courses under Contrast 6}

It was mentioned at the beginning of this subsection that there are activations observed under contrast 6 in BA19, in anterior cingulate, and in BA10. These are expected to emphasize processing of the static stimuli. This can be checked by considering the relevant time courses. That in cingulate turns out to be almost identical to that of Fig. 3e (so will not be shown), due to the high activations during the putative MAE periods (DO and BO), which is included in the static period (from the contrasts under Data Analysis). However the time courses in BA19 and in BA10 are different. That for BA19 is shown in Fig. 3f.

As seen from the figure the time course involves activations during only the static periods. There is about the same activation during the total static period (MAE + Rest) after the DO motion compared to that after the BO motion, as well as much lower but roughly equal activation during the latter motion compared to the former. Thus this site is processing static stimulus characteristics.

The activation time course of BA10 is very similar to that of other frontal regions, such as cingulate and BA44 shown above. There is a common time development of the frontal regions; they have strong activations during both MAE-DO and MAE-BO periods.

\section{Conclusions on Group Studies}

It is seen from the above that the active brain regions are responding in several different ways:

(1) They respond mainly to static activation periods, as picked out by contrast 6 , such as BA19, for example.

(2) They respond mainly to motion of either form (BO or DO), such as MT.

(3) They respond mainly during the MAE periods (MAE-DO and MAE-BO), such as CG, BA44, or BA40L, being picked out by contrast 4 and not involving much activation during the rest period, in comparison to regions under class 1 . It can be seen from Fig. 2 that classes 1 and 3 are distinct.

(4) They respond mainly to the MAE DO, such as BA40R or BA37.

It is clear that a network of areas is involved in each 
of the different tasks singled out by the different responses noted above: $\mathrm{T} 1$, representing static stimuli; T2, representing moving stimuli; T3, checking (and possibly reporting) MAE; and T4, experiencing MAE.

It is not clear that these tasks are independent, since checking and reporting on MAE can also be involved in the experience of MAE. Nor are checking and reporting necessarily the same, although they have been included together since our data for the response of type 3 above are not sensitive to such a distinction. There may also be variations of attentional control across the conditions, since there is the need to suppress smooth pursuit during the motion DO and motion BO periods not present at other times. These we cannot control for and so will have been included in activations in some regions. However, there is no relative drift of the cross and background during the MAE period. The MAE is experienced as a peripheral "bending" of the static bars during the MAE-DO period; this period may not have the same problem of the presence of other processing occurring.

\section{NETWORKS INVOLVED IN MAE}

From the individual subject time courses (not reported here in detail) motion sensitivity of MT as well as of temporoparietal regions was detected in all subjects. MAE was also seen both in MT and in a network of posterior and anterior sites, which varied somewhat between subjects. However, there was consistent use in most subjects of anterior cingulate and BA47, as well as BA40. These regions are therefore putative candidates for supporting awareness of MAE. In order to develop a more detailed analysis we need to determine how well connected these various sites are during the processing. We do that by determining the correlation coefficients between the various regions as arising from the group study time courses.

The correlation matrix between the various relevant areas is given in Table 3 . This shows that the different brain sites are broadly divisible into two different networks, one of posterior sites involved mainly with the stimuli involving motion and the anterior sites with checking and experience of the MAE itself.

The two networks are seen from Table 3 to be a posterior network, BA17/18/19/MT, and an anterior network, BA37/40/44/46/47/CG, in which we have included BA37 and BA40 because of the similarity of the time courses of their activities to the other anterior sites compared to the posterior sites. The most posterior of the sites with candidacy for belonging to the anterior network is BA37; that appears, however, to be outside MT, according to the range of coordinates given by Tootell et al. (1995b): the site in BA37 is at $(60,-56$, $-4)$, which is more than 2 standard deviations from the position $(45,-76,-3)$ as given in that reference. We have evidence to support this assignment from a more detailed analysis of the time courses, using autocorrelation functions, which distinguish this site in BA37 functionally from the MT sites (Taylor et al., 1999). The site, which we denote BA37, may be a bridge between the more anterior and the posterior networks. The natures of the two networks are shown in Fig. 4.

We conclude from Fig. 4 that there are two somewhat independent networks in the cortex which are involved in the total experience. The posterior network is activated more strongly during the motion periods and can be regarded as acting as a preprocessing net for the anterior. This can be interpreted as involved in checking and related operations most crucially during the MAE period and during static stimulus input.

\section{SUMMARY AND DISCUSSION}

We have presented a set of time courses for the activation of different areas of the cortex obtained from a study of nine subjects experiencing the MAE while they were being imaged by fMRI at $1.5 \mathrm{~T}$. We used a whole-head coverage of the slice positions so that all regions of cortex were imaged (although we did not include the cerebellum). The timing of the activations in different regions was examined by means of a group study (and analysis of single subjects was also done but not reported here due to lack of space). The group study led to a clear picture of the nature of the activations in the different cortical sites and in particular of their linkage to the timing of the various components of the stimulus input paradigm used. Motion and motion sensitivity, including response during the motion aftereffect period, were found to be concentrated in posterior sites, mainly in MT. The sensitivity to the MAE period as well as to that when static bars were input was found more anterior. The two distinct forms of response divided the cortex into two networks, one consisting of BA 18/19/39, including MT, and one containing BA 37/40/44/46/47/CG. These two networks have relatively low coupling between them, as measured by the mutual cross-correlation coefficients.

A separate study (W. Fillenz, N. Schmitz, and J. G. Taylor, unpublished) showed that the use of independent component analysis in the group study leads to two separate independent components, as well as to noise terms. These components appear to divide the cortical sites in the same manner as the networks of Fig. 4. Thus it would appear as if the networks were acting somewhat independent of each other. This is supported by consideration of the autocorrelation and partial autocorrelation functions of the time courses of the different regions; again they support a similar subdivision of the various sites (Taylor et al., 1999).

So far we have analyzed the data in order to detect the nature of processing that occurs. Our findings 
TABLE 3

Pearson Correlation Coefficients between the Sites of Activation in the MAE, for the Group Study from the Regions in Table 2

\begin{tabular}{|c|c|c|c|c|c|c|}
\hline \multirow{2}{*}{$\begin{array}{c}\text { Pearson } \\
\text { correlation }\end{array}$} & \multicolumn{6}{|c|}{ Correlations } \\
\hline & BA37 & BA40C4 & BA40CL4 & BA44C4 & BA44LC4 & BA46C4 \\
\hline BA17C1 & $0.231^{*}$ & $-0.275^{* *}$ & $-0.194^{*}$ & 0.075 & 0.147 & -0.135 \\
\hline BA17LC1 & $0.413^{* *}$ & 0.032 & -0.016 & $0.293^{* *}$ & $0.276^{* *}$ & 0.132 \\
\hline BA18C1 & 0.033 & $-0.243^{* *}$ & $-0.244^{* *}$ & -0.085 & 0.104 & -0.106 \\
\hline BA18LC1 & 0.180 & -0.148 & $-0.199^{*}$ & $0.206^{*}$ & $0.183^{*}$ & -0.113 \\
\hline BA19C1 & 0.110 & $-0.199 *$ & $-0.276^{* *}$ & 0.102 & $0.224^{*}$ & -0.092 \\
\hline BA19LC1 & -0.131 & $-0.240 * *$ & $-0.408^{* *}$ & 0.040 & 0.098 & $-0.286^{* *}$ \\
\hline BA37 & 1.000 & $0.384^{* *}$ & $0.544^{* *}$ & $0.433^{* *}$ & $0.473^{* *}$ & $0.616^{* *}$ \\
\hline BA40C4 & $0.384 * *$ & 1.000 & $0.540^{* *}$ & $0.480 * *$ & $0.658 * *$ & $0.646^{* *}$ \\
\hline BA40CL4 & $0.544 * *$ & $0.540 * *$ & 1.000 & $0.589 * *$ & $0.524 * *$ & $0.732^{* *}$ \\
\hline BA44C4 & $0.433 * *$ & $0.480 * *$ & $0.589 * *$ & 1.000 & $0.714^{* *}$ & $0.513^{* *}$ \\
\hline BA44LC4 & $0.473 * *$ & $0.658 * *$ & $0.524 * *$ & $0.714^{* *}$ & 1.000 & $0.590 * *$ \\
\hline $\mathrm{BA} 46 \mathrm{C} 4$ & $0.616^{* *}$ & $0.646^{* *}$ & $0.732^{* *}$ & $0.513^{* *}$ & $0.590^{* *}$ & 1.000 \\
\hline BA47C4 & $0.493^{* *}$ & $0.333^{* *}$ & $0.460 * *$ & $0.662 * *$ & $0.492 * *$ & $0.431^{* *}$ \\
\hline CGC4 & $0.467 * *$ & $0.577 * *$ & $0.774^{* *}$ & $0.660 * *$ & $0.485^{* *}$ & $0.767^{* *}$ \\
\hline MOBA44 & $0.521 * *$ & $0.642 * *$ & $0.668^{* *}$ & $0.717 * *$ & $0.738 * *$ & $0.691^{* *}$ \\
\hline MOBA44L & $0.622 * *$ & $0.282 * *$ & $0.564 * *$ & $0.612 * *$ & $0.495^{* *}$ & $0.494^{* *}$ \\
\hline MOCG & $0.654 * *$ & $0.437 * *$ & $0.748^{* *}$ & $0.558^{* *}$ & $0.481 * *$ & $0.712^{* *}$ \\
\hline MTL & $0.418 * *$ & 0.063 & 0.014 & $0.264 * *$ & $0.397 * *$ & $0.191^{*}$ \\
\hline \multirow[t]{2}{*}{ MTR } & $0.467 * *$ & -0.114 & 0.080 & $0.300 * *$ & $0.311^{* *}$ & 0.126 \\
\hline & BA17C1 & BA17LC1 & BA18C1 & BA18LC1 & BA19C1 & BA19LC1 \\
\hline BA17C1 & 1.000 & $0.716^{* *}$ & $0.441^{* *}$ & $0.788^{* *}$ & $0.754^{* *}$ & $0.640^{* *}$ \\
\hline BA17LC1 & $0.716^{* *}$ & 1.000 & $0.278^{* *}$ & $0.645^{* *}$ & $0.650 * *$ & $0.434^{* *}$ \\
\hline BA18C1 & $0.441^{* *}$ & $0.278 * *$ & 1.000 & $0.475^{* *}$ & $0.634^{* *}$ & $0.640^{* *}$ \\
\hline BA18LC1 & $0.788 * *$ & $0.645^{* *}$ & $0.475^{* *}$ & 1.000 & $0.646^{* *}$ & $0.636^{* *}$ \\
\hline BA19C1 & $0.754^{* *}$ & $0.650 * *$ & $0.634^{* *}$ & $0.646^{* *}$ & 1.000 & $0.780 * *$ \\
\hline BA19LC1 & $0.640 * *$ & $0.434^{* *}$ & $0.640^{* *}$ & $0.636^{* *}$ & $0.780^{* *}$ & 1.000 \\
\hline BA37 & $0.231^{*}$ & $0.413 * *$ & 0.033 & 0.180 & 0.110 & -0.131 \\
\hline BA40C4 & $-0.275^{* *}$ & 0.032 & $-0.243^{* *}$ & -0.148 & $-0.199 *$ & $-0.240 * *$ \\
\hline BA40CL4 & $-0.194^{*}$ & -0.016 & $-0.244^{* *}$ & $-0.199^{*}$ & $-0.276^{* *}$ & $-0.408^{* *}$ \\
\hline BA44C4 & 0.075 & $0.293 * *$ & -0.085 & $0.206^{*}$ & 0.102 & 0.040 \\
\hline BA44LC4 & 0.147 & $0.276 * *$ & 0.104 & $0.183^{*}$ & $0.224^{*}$ & 0.098 \\
\hline BA46C4 & -0.135 & 0.132 & -0.106 & -0.113 & -0.092 & $-0.286^{* *}$ \\
\hline BA47C4 & $0.292 * *$ & $0.466^{* *}$ & -0.054 & $0.382^{* *}$ & 0.100 & 0.010 \\
\hline CGC4 & $-0.220 *$ & 0.066 & $-0.392^{* *}$ & -0.151 & $-0.265^{* *}$ & $-0.421^{* *}$ \\
\hline MOBA44 & 0.048 & $0.316^{* *}$ & -0.128 & 0.121 & -0.040 & -0.109 \\
\hline MOBA44L & $0.209^{*}$ & $0.498 * *$ & -0.041 & $0.185^{*}$ & 0.181 & -0.155 \\
\hline MOCG & 0.106 & $0.335^{* *}$ & -0.133 & 0.078 & 0.019 & -0.151 \\
\hline MTL & $0.597 * *$ & $0.648^{* *}$ & $0.619 * *$ & $0.563^{* *}$ & $0.717 * *$ & $0.615^{* *}$ \\
\hline MTR & $0.521 * *$ & $0.506^{* *}$ & $0.628 * *$ & $0.553 * *$ & $0.636^{* *}$ & $0.469 * *$ \\
\hline
\end{tabular}

support the proposal that there is a checking/reporting process performed by the anterior network applied to output arising from the posterior network. The links between the two networks, the regions BA37 and BA40, have intermediate, but different, sensitivities to the motion and to the MAE/static periods. We suggest that the correlation decomposition has separated the processing into the two sources of information, one arising from the internal task set by the subject (that of determining how long the MAE occurs for them) and the other from the input of the moving/static bars and the fixation cross. Such a decomposition must also include components arising from an attentional/fixation network as well as one preserving a general level of vigilance. These involve mainly right-hemisphere cortical sites. We cannot disentangle these here since we did not use different levels of attentional processing for the task by providing distractors at various levels.

We have thus attained the two main purposes we outlined at the beginning of the paper: to replicate the results of Tootell et al. (1995a) and to search for the total network involved in the MAE. We are still left with the problem of assessing the roles played by the various areas in the phenomenal experience of the MAE, as guided by our experimental results. Let us turn to this question now. We use the result of He et al. (1998) that activity in MT is not sufficient to produce the MAE experience itself. The methods used by He et 
TABLE 3-Continued

\begin{tabular}{|c|c|c|c|c|c|c|c|}
\hline \multirow{2}{*}{$\begin{array}{c}\text { Pearson } \\
\text { correlation }\end{array}$} & \multicolumn{7}{|c|}{ Correlations } \\
\hline & BA47C4 & CGC4 & MOBA44 & MOBA44L & MOCG & MTL & MTR \\
\hline BA17C1 & $0.292 * *$ & $-0.220^{*}$ & 0.048 & $0.209 *$ & 0.106 & $0.597^{* *}$ & $0.521^{* *}$ \\
\hline BA17LC1 & $0.466^{* *}$ & 0.066 & $0.316^{* *}$ & $0.498 * *$ & $0.335^{* *}$ & $0.648^{* *}$ & $0.506^{* *}$ \\
\hline BA18C1 & -0.054 & $-0.392^{* *}$ & -0.128 & -0.041 & -0.133 & $0.619 * *$ & $0.628^{* *}$ \\
\hline BA18LC1 & $0.382^{* *}$ & -0.151 & 0.121 & $0.185^{*}$ & 0.078 & $0.563^{* *}$ & $0.553^{* *}$ \\
\hline BA19C1 & 0.100 & $-0.265^{* *}$ & -0.040 & 0.181 & 0.019 & $0.717 * *$ & $0.636^{* *}$ \\
\hline BA19LC1 & 0.010 & $-0.421^{* *}$ & -0.109 & -0.155 & -0.151 & $0.615^{* *}$ & $0.469^{* *}$ \\
\hline BA37 & $0.493^{* *}$ & $0.467^{* *}$ & $0.521^{* *}$ & $0.622^{* *}$ & $0.654^{* *}$ & $0.418^{* *}$ & $0.467^{* *}$ \\
\hline BA40C4 & $0.333^{* *}$ & $0.577 * *$ & $0.642^{* *}$ & $0.282^{* *}$ & $0.437 * *$ & 0.063 & -0.114 \\
\hline BA40CL4 & $0.460 * *$ & $0.774^{* *}$ & $0.668^{* *}$ & $0.564^{* *}$ & $0.748^{* *}$ & 0.014 & 0.080 \\
\hline $\mathrm{BA} 44 \mathrm{C} 4$ & $0.662^{* *}$ & $0.660 * *$ & $0.717^{* *} *$ & $0.612^{* *}$ & $0.558 * *$ & $0.264^{* *}$ & $0.300^{* *}$ \\
\hline BA44LC4 & $0.492 * *$ & $0.485^{* *}$ & $0.738^{* *}$ & $0.495^{* *}$ & $0.481^{* *}$ & $0.397 * *$ & $0.311^{* *}$ \\
\hline $\mathrm{BA} 46 \mathrm{C} 4$ & $0.431^{* *}$ & $0.767^{* *}$ & $0.691^{* *}$ & $0.494 * *$ & $0.712^{* *}$ & $0.191 *$ & 0.126 \\
\hline BA47C4 & 1.000 & $0.513^{* *}$ & $0.611 * *$ & $0.512^{* *}$ & $0.545^{* *}$ & $0.353^{* *}$ & $0.366^{* *}$ \\
\hline CGC4 & $0.513 * *$ & 1.000 & $0.682^{* *}$ & $0.535^{* *}$ & $0.683^{* *}$ & -0.065 & -0.005 \\
\hline MOBA44 & $0.611^{* *}$ & $0.682 * *$ & 1.000 & $0.619 * *$ & $0.609 * *$ & $0.318^{* *}$ & $0.193^{*}$ \\
\hline MOBA44L & $0.512^{* *}$ & $0.535^{* *}$ & $0.619 * *$ & 1.000 & $0.667 * *$ & $0.353^{* *}$ & $0.345^{* *}$ \\
\hline MOCG & $0.545^{* *}$ & $0.683^{* *}$ & $0.609 * *$ & $0.667 * *$ & 1.000 & $0.302^{* *}$ & $0.202^{*}$ \\
\hline MTL & $0.353^{* *}$ & -0.065 & $0.318^{* *}$ & $0.353 * *$ & $0.302 * *$ & 1.000 & $0.773 * *$ \\
\hline MTR & $0.366^{* *}$ & -0.005 & $0.193^{*}$ & $0.345^{* *}$ & $0.202^{*}$ & $0.773^{* *}$ & 1.000 \\
\hline
\end{tabular}

** Correlation is significant at the 0.01 level (two-tailed).

* Correlation is significant at the 0.05 level (two-tailed).

al. (1998), when applied to a whole-head measurement, could settle the question of which regions are sufficient for such a task.

Until that answer is available we will try to give a tentative answer from our admittedly incomplete paradigm. We consider the time courses of the various anterior areas. These differ, with some sites having more sensitivity to the motion period, some being more sensitive to the static period, others mainly sensitive to the MAE-DO period, and yet others sensitive to both MAE periods. The sites mediating most uniquely the MAE-DO experience, from Fig. 3 (and from other data we have taken, unpublished), are BA40 and BA37, with some involvement of BA44. BA40 is the site of various working memory/multimodal/

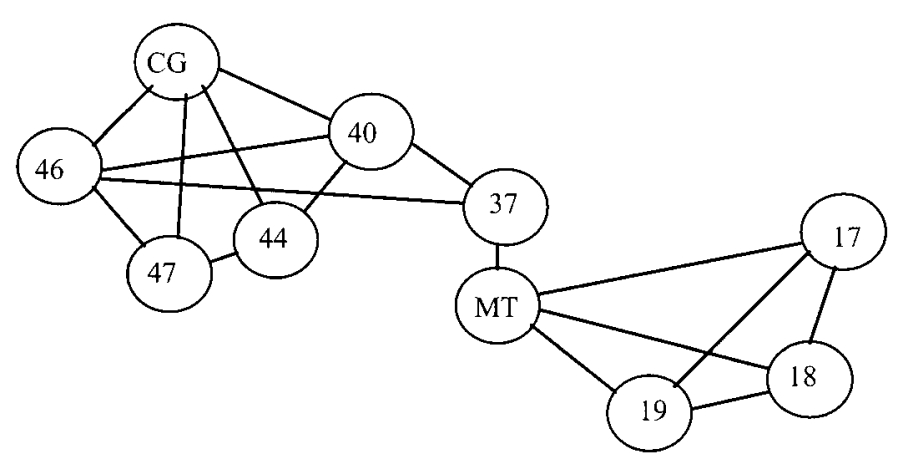

FIG. 4. The correlation graph of the active cortical areas for MAE, as deduced from the correlations of Table 3 . We use the notation CG for cingulate gyrus. The lines join cortical sites which have cross correlations of at least 0.5 . attentional modules (Coull and Nobre, 1998; Jonides et al., 1998) and so would agree with conjectures (Newell and Simon, 1972; Taylor, 1992) that the creation of awareness crucially involves such a site. The involvement of the other sites mentioned above is still uncertain. This question is discussed in more detail by us elsewhere (Taylor et al., 1999). In any case the data do not favor solely early visual cortical sites (Pollen, 1999) nor the solely frontally based option of Crick and Koch (1998). If anything the network reported above indicates that the creation of consciousness is more distributed, involving interaction between a number of key modules.

This still leaves unanswered the question as to where awareness is sited in the cortex outside the MAE period. We conjecture that the sites we are observing to be active solely during the MAE period are involved in an inner monitoring process similar to that observed during the concept shifts in binocular rivalry (Lumer and Rees, 1999) and on strategy shifting in the go-no go task (Konishi et al., 1999); distributed networks which both include the inferior parietal lobe are activated in both these tasks, which are correlated to the time of shift. Other components of the network are different, lending support to the possible identification of the inferior parietal lobe with a higher order monitoring/attentional system advocated by some (Armstrong, 1968; Rosenthal, 1986) as at the basis of consciousness. From this approach sites for nonmonitoring consciousness are active during other periods; other paradigms are needed to make them conspicuous. 


\section{ACKNOWLEDGMENTS}

One of us (J.G.T.) thanks the successive Directors (Professors H.-W. Mueller-Gaertner and K. Zilles) for hospitality at the Institute for Medicine, Fz-Juelich, Germany, where the work was performed.

\section{REFERENCES}

Armstrong, D. M. 1968. A Materialist Theory of Mind. Routledge \& Kegan Paul, London.

Adams, R. 1984. Philosophical Magazine 5:373-574.

Coull, J. T., and Nobre, A. C. 1998. Where and when to pay attention: The neural systems for directing attention to spatial locations and to time intervals as revealed by both PET and fMRI. J. Neurosci. 18:7426-7435.

Crick, F. H. C., and Koch, C. 1998. Consciousness and neuroscience. Cereb. Cortex 8:97-107.

Friston, K. J., Holmes, A. P., Worsley, K. J., Poline, C.-D., Frith, C. D., and Frakowiak, S. J. 1995. Statistical parametric maps in functional imaging: A general linear approach. Hum. Brain Mapp. 2:189-210.

Friston, K. J., Jezzard, P., and Turner, R. 1994. Analysis of functional MRI time-series. Hum. Brain Mapp. 1:153-171.

He, S., Cohen, E. R., Costello, P. A., Yacoub, E., and Hu, X. 1998. NeuroImage 7:S326.

Jonides, J., Shumacher, E. H., Smith, E. E., Koeppe, R. A., Awh, E., Reuter-Lorenz, O. A., Marshuetz, C., and Willis, C. R. 1998. The role of parietal cortex in verbal working memory. J. Neurosci. 18:5026-5034.

Konishi, S., Nakajima, K., Uchida, I., Kikyo, H., Kameyama, M., and Miyashita, Y. 1999. Common inhibitory mechanisms in human inferior prefrontal cortex revealed by event-related functional fMRI. Brain 122:981-991.

Lumer, E. D., and Rees, G. 1999. Covariation of activity in visual and prefrontal cortex associated with subjective visual perception. Proc. Natl. Acad. Sci. USA 96:1669-1673.

Mather, G., Verstraten, F., and Anstis, S. 1998. The Motion Aftereffect. MIT Press, Cambridge, MA.

MEL Professional. 1996. Version 2.0. Psychology Software Tools Inc., Pittsburgh, PA.

Moutoussis, K., and Zeki, S. 1997. Functional segregation and temporal hierarchy of the visual perceptive systems. Proc. R. Soc. London B 264:1407-1414.

Newell, A., and Simon, H. A. 1972. Human Problem Solving. Prentice Hall, Englewood Cliffs, NJ.

Pollen, D. 1999. On the neural correlates of visual perception. Cereb. Cortex, 9:4-19.

Rosenthal, D. M. 1986. Two concepts of consciousness. Philos. Stud. 94:329-359.

Talairach, J., and Tournoux, P. 1988. Co-planar Stereotaxic Atlas of the Human Brain. Thieme, Stuttgart.

Taylor, J. G. 1992. Towards a neural network model of the mind. Neural Network World 2:797-812.

Taylor, J. G. 1996. A competition for consciousness? NeuroComputing 11:271-296.

Taylor, J. G. 1997. Neural networks for consciousness. Neural Networks 10:1207-1225.

Taylor, J. G., Schmitz, N., Ziemons, K., Gruber, O., Grosse-Ruyken, M.-L., and Shah, N. J. 1999. Awareness and the motion aftereffect. In preparation.

Tootell, R. B. H., Reppas, J. B., Dale, A. M., Look, R. B., Sereno, M. I., Malach, R., Brady, T. J., and Rosen, B. R. 1995a. Visual motion aftereffect in human cortical area MT revealed by functional magnetic resonance imaging. Nature 375:139-141.

Tootell, R. B. H. et al. 1995b. J. Neurosci. 15:3215-3230.

Wohlegemuth, A. 1911. Br. J. Psychol. Suppl. 1:1-117. 